\title{
Open Wedge High Tibial Osteotomy and Combined Arthroscopic Surgery in Severe Medial Osteoarthritis and Varus Malalignment: Minimum 5-Year Results
}

\author{
Moon-Jib Yoo, MD and Yong-Eun Shin, MD \\ Department of Orthopedic Surgery, Dankook University Hospital, Dankook University School of Medicine, Cheonan, Korea
}

\begin{abstract}
Purpose: To evaluate the radiologic and functional outcomes of medial open wedge high tibial osteotomy (HTO) combined with arthroscopic procedure in patients with medial osteoarthritis.

Materials and Methods: From June 1996 to March 2010, 26 patients (32 knees) who underwent medial open wedge osteotomy and arthroscopic operation for medial osteoarthritis were retrospectively reviewed. Measurements included hip-knee-ankle (HKA) angle, femorotibial angle, medial proximal tibial angle, posterior tibial slope angle, and Kellgren-Lawrence grade. Clinical evaluation was performed using Lysholm knee scoring scale and knee and function score of the American Knee Society.

Results: Differences between the mean preoperative and postoperative measurements were significant in all angles including the HKA angle $\left(-5.7^{\circ}\right.$ and $\left.+5.5^{\circ}\right)$, femorotibial angle $\left(-1.9^{\circ}\right.$ and $\left.+9.8^{\circ}\right)$, and medial proximal tibial angle $\left(82.9^{\circ}\right.$ and $\left.90.5^{\circ}\right)(\mathrm{p}<0.05)$. Mean Lysholm knee scoring scale was 63.6 preoperatively and 88.7 at the last follow-up, mean Knee Society knee score was 61.2 and 86.6 , and mean function score was 59.3 and 87.2 , respectively. All differences were significant $(\mathrm{p}<0.05)$.

Conclusions: Medial open wedge HTO in combination with arthroscopic procedure is an effective treatment method for medial osteoarthritis to treat varus deformity and an intra-articular lesion.
\end{abstract}

Keywords: Knee, Osteoarthritis, Osteotomy, Arthroscopy

\section{Introduction}

The average age and life expectancy have increased in Korea due to the aging of the society. Accordingly, the incidence of degenerative arthritis has increased sharply ${ }^{1)}$. Degenerative knee arthritis is one of the most common diseases in the elderly.

There are many treatment options for degenerative arthritis.

Received December 21, 2015; Accepted February 22, 2016

Correspondence to: Moon-Jib Yoo, MD

Department of Orthopaedic Surgery, Dankook University Hospital,

Dankook University Medical College, 201 Manghyang-ro, Dongnam-gu, Cheonan 31116, Korea

Tel: +82-41-550-6290, Fax: +82-41-550-6319

E-mail: mjyoo@dankook.ac.kr

Source of funding: This research was conducted by the research fund of Dankook University in 2015.

This is an Open Access article distributed under the terms of the Creative Commons Attribution Non-Commercial License (http://creativecommons.org/licenses/by-nc/4.0/) which permits unrestricted non-commercial use, distribution, and reproduction in any medium, provided the original work is properly cited.
Conservative treatments include medication and physical therapy. Surgical treatments include arthroscopic operation, corrective osteotomy, and knee replacement arthroplasty. It is important to select an adequate treatment option considering patient age, level of activity, lower extremity alignment, and body mass index ${ }^{2,3)}$.

Proximal tibial osteotomy has been used to treat knee joint degenerative arthritis with varus deformity since first described in 1960 by Jackson and Waugh ${ }^{4}$. Although subsequently refined ${ }^{5}$, it has more recently become less popular because of the development of total knee replacement arthroplasty (TKRA) $)^{6,7)}$. In spite of the increasing use of TKRA, proximal tibial osteotomy is still a primary choice in active, relatively young knee osteoarthritis patients whose lesions are limited to the medial compartment, because the approach can slow the progress of degeneration, relieve pain, and correct lower extremity alignment ${ }^{1,8)}$.

In the present study, we retrospectively examined the longterm clinical results of combined proximal tibial osteotomy and arthroscopic operation in the knee joint degenerative arthritis. Clinical and radiologic results of the patients were studied. We 
hypothesized that the outcomes of proximal tibial osteotomy combined with arthroscopic operation would be beneficial for the treatment of medial osteoarthritis and intra-articular lesions for relatively young patients.

\section{Materials and Methods}

\section{Subjects}

After approval of our Institutional Review Board, we retrospectively assessed 26 patients ( 32 knees) that had been followed for at least 5 years. The patients underwent proximal tibial medial open wedge osteotomy and arthroscopy due to knee joint degenerative arthritis by the senior author from June 1996 to March 2010. The 26 patients comprised 8 males and 18 females, with a mean age of 49.3 years (range, 41 to 60 years) at the time of surgery. The mean follow-up duration was 8 years and 9 months (range, 5 years 4 months to 12 years 10 months) (Table 1). Surgical indications were age under 60, Kellgren-Lawrence grade ${ }^{9)}$ III or IV in which lesions were limited to the medial compartment, malalignment with a genu varum (hip-knee-ankle [HKA] angle passed through the medial tibial plateau or was more than $-3^{\circ}$ compared to the opposite site), no general laxity, flexion contracture under $15^{\circ}$. Patients with rheumatoid arthritis, history of treatment for fracture around the knee joint, or a lesion in the patellofemoral joint or lateral compartment of femorotibial joint were excluded.

\section{Surgical Procedure}

The HKA and femorotibial angles were assessed using the preoperative weight bearing anteroposterior plain radiographs. The ideal postoperative HKA angle and femorotibial angle were considered as valgus $3^{\circ}-5^{\circ}$ and valgus $8^{\circ}-10^{\circ}$, respectively. An arthroscopic microfracture was done if there were International

Table 1. Demographics

\begin{tabular}{lc}
\hline \multicolumn{1}{c}{ Variable } & Mean (range) \\
\hline Age (yr) & $49.3(41-60)$ \\
Gander (M:F) & $8: 18$ \\
Preoperative symptom duration (mo) & $39(0.5-180)$ \\
Follow-up duration (mo) & $105(64-154)$ \\
Arthroscopic procedure (overlap) & \\
Microfracture & 24 cases \\
Meniscectomy & 18 cases \\
Plica excision & 4 cases \\
Body mass index $\left(\mathrm{kg} / \mathrm{m}^{2}\right)$ & $25.9(22.3-31.3)$ \\
Average operation time (min) & $67.2(57-85)$ \\
\hline
\end{tabular}

Cartilage Repair Society grade 3 or 4 lesions in the femoral or tibial articular cartilage. Arthroscopic partial meniscectomy was performed for medial meniscus tears. Other procedures including loose body removal, plica excision, and synovectomy were done. Position change to Trendenlenburg $10^{\circ}$ after arthroscopic procedure and longitudinal skin incision were done about $5 \mathrm{~cm}$ distal to the medial joint line, perpendicular to the pes anserinus. Proximal to the pes anserinus, superficial medial collateral ligament was dissected off the posteromedial cortex of the tibia. After soft tissue dissection, a blunt Hohmann retractor was inserted posteriorly to protect posterior neurovascular structures. A guide wire was inserted $2.5-3 \mathrm{~cm}$ below the medial joint line to the area between the tip of the fibular head and the circumference line of the fibular head (the safe zone) (Fig. 1) ${ }^{10)}$. The location of guide wire was examined by a fluoroscope. The osteotomy was performed just above the guide wire using an oscillating saw, and an osteotome chisel was placed into the osteotomy site. During osteotomy, care should be taken to ensure the osteotomy line extends $1 \mathrm{~cm}$ medial to the lateral cortex to avoid lateral cortical fracture ${ }^{10)}$ and is parallel to posterior tibial slope on the sagittal plane. After opening the osteotomy site using a bone spreader, an electronic coagulator was aligned from the center of the hip joint to the center of the ankle joint to pass $62 \%$ lateral to the tibial plateau (Fujisawa point ${ }^{11}$. The site was fixed using a Chang tibia osteotomy plate system (C\&S Medical, Seoul, Korea), and a demineralized chip bone allograft was applied at the open wedge osteotomy site. Finally, alignment of the lower extremity was checked using fluoroscope $\mathrm{e}^{12)}$.

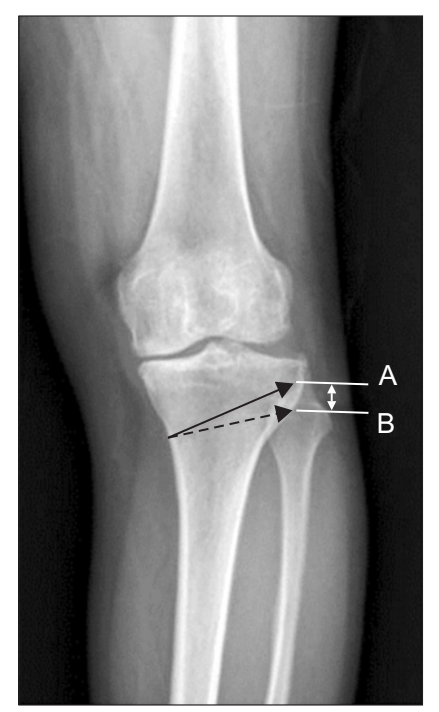

Fig. 1. Safe zone: between A and B. A: tip of fibular head. B: circumference line of the fibular head. 


\section{Clinical and Radiologic Evaluation}

Preoperative and postoperative Kellgren-Lawrence grade, HKA angle, femorotibial angle, medial proximal tibial angle, and posterior tibial slope angle were measured by plain radiography. Degenerative arthritis was evaluated by the degree of joint space narrowing, osteophytes, and subchondral sclerosis on weight bearing anteroposterior plain radiographs using KellgrenLawrence grade ${ }^{9)}$. HKA angle was measured as described by Moreland et al. ${ }^{13)}$ using weight bearing whole lower extremity anteroposterior scanograms. The acute angle subtended between the line from the center of the femoral head to the center of both femoral condyles, and the line from the center of the tibial plafond to the center of both tibial condyles was defined as the HKA angle. A negative angle indicated genu varum and a positive angle indicated genu valgum (Fig. 2). The femorotibial angle was measured as described by Bauer et al. ${ }^{14)}$ on weight bearing anteroposterior radiographs. The femorotibial angle was defined as the acute angle subtended by the long axis line from the center of femoral diaphysis $10 \mathrm{~cm}$ above the joint line to the center of

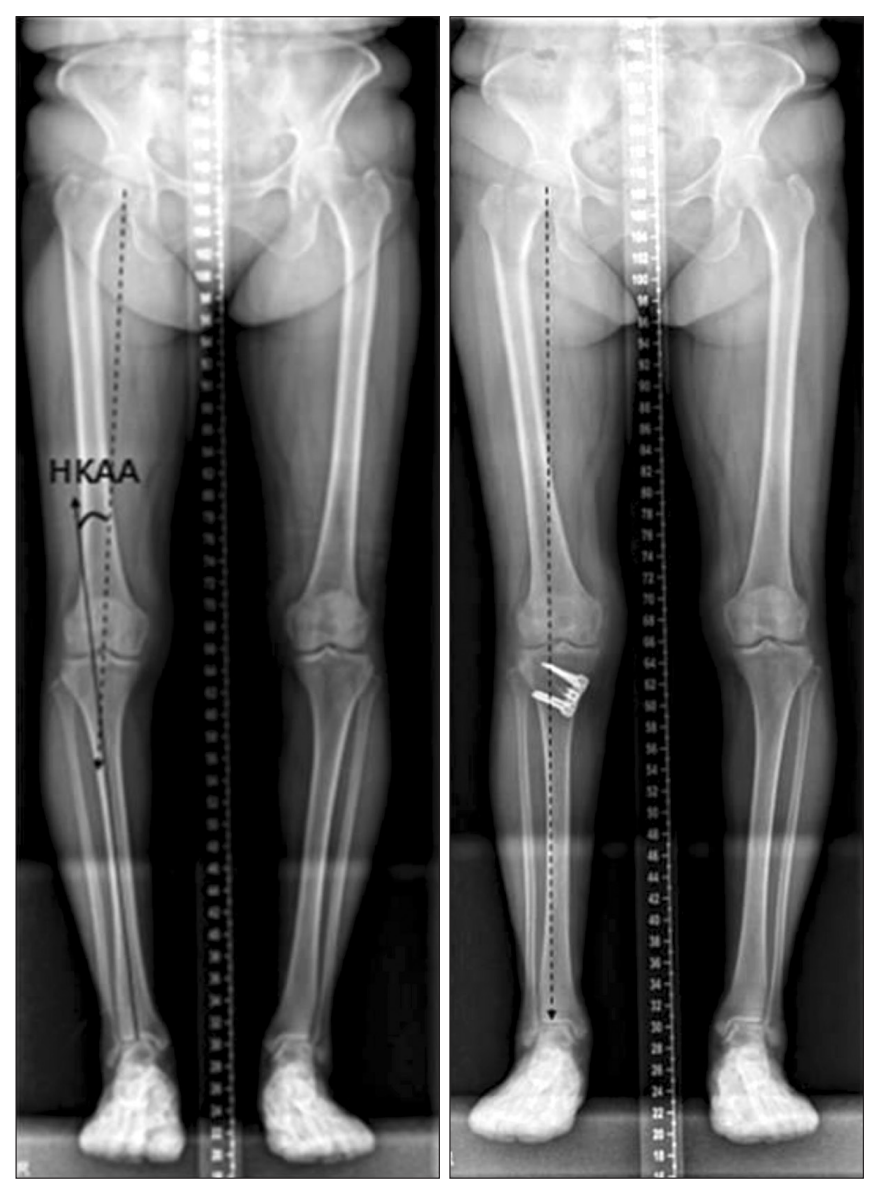

Fig. 2. Hip-knee-ankle angle (HKAA) measurement method and correction of varus deformity. Note that dash line passes the 'Fujisawa point'. femoral diaphysis $4 \mathrm{~cm}$ above the joint line and the long axis line from the center of tibial diaphysis $10 \mathrm{~cm}$ below the joint line to the center of tibial diaphysis $4 \mathrm{~cm}$ below the joint line. A negative angle indicated genu varum and a positive angle indicated genu valgum (Fig. 3). The medial proximal tibial angle was measured as the angle subtended by the long axis of tibia and a line parallel to the medial plateau on simple weight bearing knee joint anteroposterior radiographs ${ }^{15)}$ (Fig. 3). Posterior tibial slope angle was measured as described by Brandon et al. ${ }^{16)}$ on simple knee joint lateral radiographs. It was defined as the angle subtended between the tibial anatomical long axis, in the line between the center of the anteroposterior diameter measured just below the tibial tuberosity and the center of the anteroposterior diameter measured $5 \mathrm{~cm}$ distal to the former, and a line drawn from the medial tibial plateau (Fig. 3). All images were evaluated using a picture archiving and communication system (PACS; Techheim, Seoul, Korea), and two different observers independently measured preoperative and postoperative images. Clinical and functional evaluations were done before surgery, 3, 6, and 12 months postoperatively, and each year thereafter. Lysholm knee scoring scale and knee and function score of the American Knee Society were used for clinical evaluation ${ }^{17)}$.

\section{Postoperative Care}

Isotonic exercise of extensor and flexor muscles was started immediately postoperatively, with early joint motion exercise encouraged. Passive joint motion exercises (continuous passive motion) with muscle strengthening exercises were carried out, and partial weight bearing with crutch ambulation was recommended until 6 weeks after surgery. After the osteotomy site was blurred by X-ray, full weight bearing ambulation was started.

\section{Statistical Analysis}

IBM SPSS ver. 21.0 (IBM Co., Armonk, NY, USA) was used for statistical verification. Continuous variables were identified with standard distribution using the Shapiro-Wilk test, and preoperative and postoperative differences were identified using Paired $t$ test. Weighted kappa coefficient was used to evaluate the interobserver reliability.

\section{Results}

Twenty four cases of articular cartilage defect of the medial femoral condyle were identified in arthroscopic examinations, and these lesions were treated by arthroscopic microfracture (Fig. 4). Eighteen cases of medial meniscus tear in the arthroscopic examinations were treated by arthroscopic partial meniscectomy. 

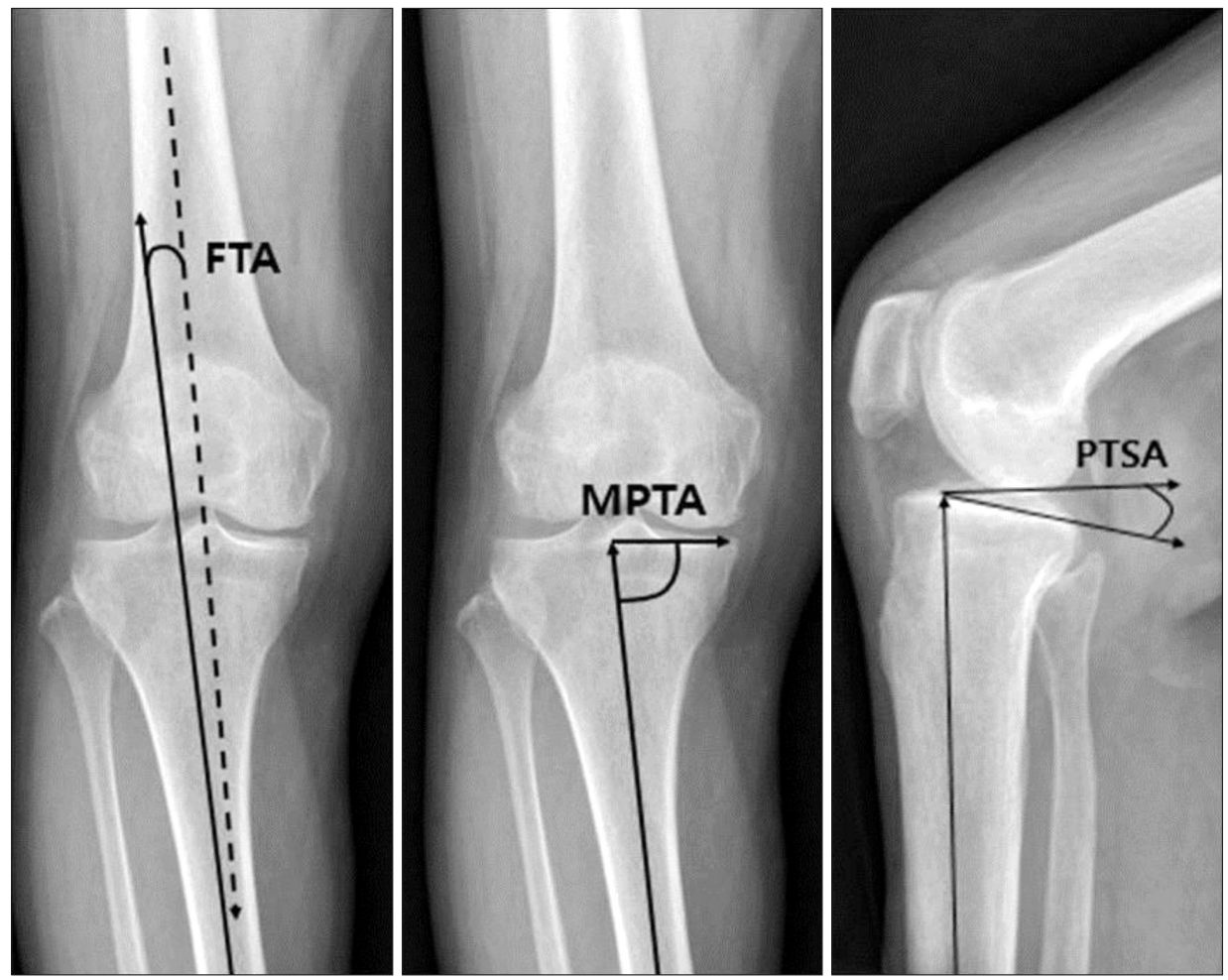

Fig. 3. Femorotibial angle (FTA), medial proximal tibial angle (MPTA), and posterior tibial slope angle (PTSA) measurement methods.
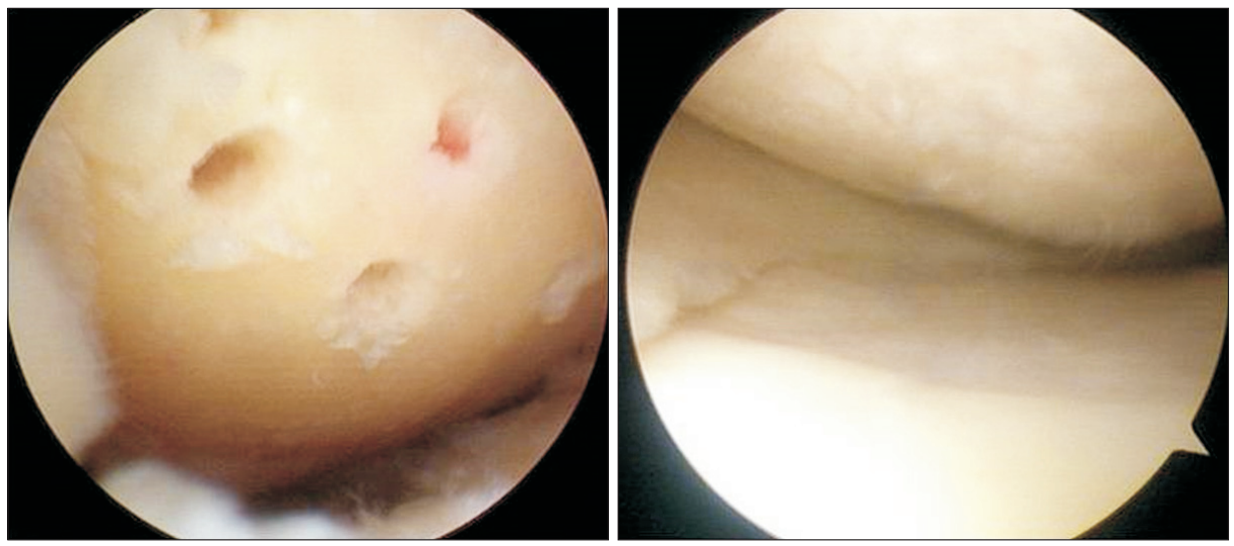

Fig. 4. (A) Osteochondral defect was detected in arthroscopy and microfracture was performed. (B) Second-look arthroscopic finding at 18 months after surgery: the articular surface was covered with fibrocartilage.

Four cases of pathologic plica identified in the arthroscopic examinations were treated by arthroscopic plica excision. The mean operation time was 67.2 minutes (Table 1).

Preoperatively, there were 28 cases of Kellgren-Lawrence grade III and 4 cases of Kellgren-Lawrence grade IV. At the last followup after surgery, there were 25 cases of Kellgren-Lawrence grade III and 7 cases of Kellgren-Lawrence grade IV. There was no significant difference between preoperation and final follow-up ( $>0.05)$.

Preoperatively and postoperatively, the mean HKA angle was $-5.7^{\circ}$ and $+5.5^{\circ}$, mean femorotibial angle was $-1.9^{\circ}$ and $+9.8^{\circ}$, and mean medial proximal tibial angle was $82.9^{\circ}$ and $90.5^{\circ}$, respec- tively (all $\mathrm{p}<0.05$ ). Mean posterior tibial slope was $6.2^{\circ}$ preoperatively and $7.3^{\circ}$ postoperatively ( $\mathrm{p}>0.05$ ) (Table 2 ).

Clinically, the mean Lysholm knee scoring scale was 63.6 preoperatively and 88.7 at the last follow-up. The mean American Knee Society score was 61.2 preoperatively and 86.6 at the last followup. The mean knee functional score was 59.3 preoperatively and 87.2 at the last follow-up. All differences were significant (Table 3). At the final follow-up, 30 of 32 cases (93.8\%) were satisfied with their symptoms.

After bone union was confirmed by radiography, the plate was removed in all cases. Six cases underwent second-look arthroscopic examination. Cases in which microfracture was performed 
Table 2. Comparison of Radiographic Results

\begin{tabular}{|c|c|c|c|}
\hline Variable & Preoperative & inal follow-up & p-value \\
\hline \multicolumn{4}{|l|}{ Kellgren-Lawrence grade (cases) } \\
\hline Grade III & 28 & 25 & 0.471 \\
\hline Grade IV & 4 & 7 & 0.121 \\
\hline Hip-knee-ankle angle $\left({ }^{\circ}\right)$ & $-5.7 \pm 1.2$ & $5.5 \pm 0.9$ & 0.013 \\
\hline Femorotibial angle $\left({ }^{\circ}\right)$ & $-1.9 \pm 0.9$ & $9.8 \pm 1.1$ & 0.012 \\
\hline Medial proximal tibial angle $\left({ }^{\circ}\right)$ & $82.9 \pm 2.1$ & $90.5 \pm 1.8$ & 0.017 \\
\hline Posterior tibial slope angle $\left({ }^{\circ}\right)$ & $6.2 \pm 1.3$ & $7.3 \pm 0.8$ & 0.319 \\
\hline
\end{tabular}

Values are presented as mean \pm standard deviation.

on the medial femoral condyle featured fibrous cartilage covering and those where partial meniscectomy was performed for medial meniscus tear were free of tears in a second-look arthroscopic examination (Fig. 4).

There were no complications including infection, nonunion, limited range of motion, metal failure and correction loss at the last follow-up. In one case, conversion to TKRA was done due to arthritic change on the lateral compartment. Interobserver reliability was in "almost perfect agreement" with a weighted kappa coefficient of 0.82 .

\section{Discussion}

TKRA cannot be the primary treatment option for relatively young patients with knee joint degenerative arthritis lesions limited to the medial compartment. Instead, proximal tibial osteotomy and unicompartment knee replacement arthroplasty (UKRA) can be viable options for medial osteoarthritis. The Korean culture of sitting on the floor induces degenerative knee arthritis and genu varum deformity ${ }^{18,19)}$. In South Korea, the incidence of proximal tibial osteotomy has recently increased from 2,649 cases in 2009 to 8,207 cases in $2013^{20)}$.

Spahn et al. ${ }^{21)}$ analyzed 46 cases of proximal tibial osteotomy and 43 cases of UKRA. They reported there was no difference in the long-term results between the two procedures in terms of clinical and radiological outcomes and complications, which led to the recommended use of proximal tibial osteotomy for patients who were relatively young, active, and have medial degenerative arthritis. Floerkemeier et al. ${ }^{22)}$ retrospectively analyzed 533 cases of proximal tibial open wedge osteotomy and reported that proximal tibial osteotomy had a better clinical outcome than UKRA in elderly patients whose articular surface was severely destroyed.

Many studies have addressed the effect of combined proximal tibial osteotomy and arthroscopic operation. Schuster et al. ${ }^{12)}$ obtained excellent radiological and clinical results after carrying
Table 3. Comparison of Clinical Results at the Final Follow-up

\begin{tabular}{lrcc}
\hline \multicolumn{1}{c}{ Variable } & Preoperative & Final follow-up & p-value \\
\hline Lysholm knee score & $63.6 \pm 8.5$ & $88.7 \pm 6.4$ & 0.013 \\
American knee score & $61.2 \pm 6.6$ & $86.6 \pm 5.9$ & 0.010 \\
American functional score & $59.3 \pm 9.2$ & $87.2 \pm 7.2$ & 0.008 \\
Range of motion $\left({ }^{\circ}\right)$ & $125.2 \pm 6.4$ & $134.8 \pm 5.2$ & 0.079 \\
\hline
\end{tabular}

Values are presented as mean \pm standard deviation.

out proximal tibial osteotomy with arthroscopic curettage and microfracture, with $94.9 \%$ of patients being satisfied. Pascale et al. ${ }^{23)}$ prospectively studied 40 cases of medial knee degenerative arthritis in two patient groups: proximal tibial osteotomy with and without arthroscopic microfracture. There were no significant differences between the two groups, but subjective satisfaction was higher in the former group at 5-year follow-up.

Some authors suggest that combined proximal tibial osteotomy and arthroscopic procedure is not effective. Jung et al. ${ }^{24)}$ retrospectively compared 30 cases of combined proximal tibial osteotomy and arthroscopic subchondral drilling and 31 cases of proximal tibial osteotomy alone. There were no differences between the two groups in terms of clinical outcome and the degree of fibrous cartilage regeneration at two years of follow-up. Therefore, they suggested that arthroscopic subchondral drilling was not effective. Matsunaga et al. ${ }^{25)}$ also reported that there was no benefit in clinical results of arthroscopic microfracture or curettage in proximal tibial osteotomy for medial osteoarthritis.

The benefit of additional arthroscopic operation in proximal tibial osteotomy is still unclear. There are several studies focusing on survival rates after high tibial osteotomy (Table 4$)^{26-30}$. Harris et al. ${ }^{26)}$ reviewed several literatures and suggested that the 5 -year survival rate of combined proximal tibial osteotomy and arthroscopic procedure was $97.7 \%$, and that of isolated proximal tibial osteotomy was $92.4 \%$. Combined proximal tibial osteotomy and arthroscopic procedure is better than isolated proximal tibial osteotomy in the aspect of survivorship; however, there are numerous differences in surgical techniques, follow-up periods, inclusion criteria, and severity of medial compartment osteoarthritis among studies. In our study, the 5-year survival rate of combined proximal tibial open wedge osteotomy and arthroscopic operation was $96.9 \%$; consequently, our results add weight to the findings described in the above studies.

Furthermore, additional arthroscopic operation in the proximal tibial osteotomy allowed evaluation of intra-articular lesions in patients who did not undergo preoperative magnetic resonance imaging and treatment of such lesions. Thus, it can be considered cost-effective. 
Table 4. Overview of Survival Rates after Proximal Tibial Osteotomy in Recent Studies

\begin{tabular}{|c|c|c|c|c|}
\hline Author & Year & $\begin{array}{l}\text { Additional } \\
\text { arthroscopic operation }\end{array}$ & $\begin{array}{l}\text { No. of } \\
\text { cases }\end{array}$ & $\begin{array}{l}\text { 5-yr survival } \\
\text { rate }(\%)\end{array}$ \\
\hline \multirow[t]{2}{*}{ Harris et al. ${ }^{26)}$} & 2013 & - & 4,396 & 92.4 \\
\hline & & $\begin{array}{l}\text { Procedure for articular } \\
\text { cartilage lesion }\end{array}$ & 399 & 97.7 \\
\hline Sterett et al. ${ }^{27)}$ & 2010 & Chondral resurfacing & 106 & 97.0 \\
\hline Hernigou et al. ${ }^{28)}$ & 2010 & - & 53 & 88.7 \\
\hline Laprade et al. ${ }^{29)}$ & 2012 & $\begin{array}{l}\text { Diagnostic arthros- } \\
\text { copy when indicated }\end{array}$ & 47 & 96.0 \\
\hline Bode et al. ${ }^{30)}$ & 2013 & - & 62 & 96.0 \\
\hline
\end{tabular}

Our study had several limitations. First, the sample size was not sufficiently large to draw conclusions. Second, the design of the study was retrospective. Third, there was no control group to compare the effects of additional arthroscopic treatment. Nevertheless, the significance of this study is that it attempted to analyze the effect of additional arthroscopic treatment in a more than 5 years of relatively long-term follow-up period.

\section{Conclusions}

Medial open wedge proximal tibial osteotomy can be considered a good treatment option for degenerative arthritis of the medial compartment of the knee. Also, additional arthroscopic surgery would be helpful to treat intra-articular lesions and to prevent progression of arthritis.

\section{Conflict of Interest}

No potential conflict of interest relevant to this article was reported.

\section{References}

1. Iorio R, Robb WJ, Healy WL, Berry DJ, Hozack WJ, Kyle RF, Lewallen DG, Trousdale RT, Jiranek WA, Stamos VP, Parsley BS. Orthopaedic surgeon workforce and volume assessment for total hip and knee replacement in the United States: preparing for an epidemic. J Bone Joint Surg Am. 2008;90:1598605.

2. Krych AJ, Carey JL, Marx RG, Dahm DL, Sennett BJ, Stuart MJ, Levy BA. Does arthroscopic knee surgery work? Arthroscopy. 2014;30:544-5.

3. Marmor L. Unicompartmental and total knee arthroplasty.
Clin Orthop Relat Res. 1985;(192):75-81.

4. Jackson JP, Waugh W. Tibial osteotomy for osteoarthritis of the knee. Proc R Soc Med. 1960;53:888.

5. Coventry MB. osteotomy of the upper portion of the tibia for degenerative arthritis of the knee: a preliminary report. J Bone Joint Surg Am. 1965;47:984-90.

6. Cho WS, Bin SI, Cheong KK, Kim JC, Kim KY. Valgus high tibial osteotomy for osteoarthritis of the knee. J Korean Orthop Assoc. 1995;30:1624-30.

7. W-Dahl A, Robertsson O, Lohmander LS. High tibial osteotomy in Sweden, 1998-2007: a population-based study of the use and rate of revision to knee arthroplasty. Acta Orthop. 2012;83:244-8.

8. Kim BJ, Ko HS, Lim Y, Seo JG, Joo SG, Suh JS, Sin JG. High tibial osteotomy. J Korean Orthop Assoc. 1994;29:627-33.

9. Kellgren JH, Lawrence JS. Radiological assessment of osteoarthrosis. Ann Rheum Dis. 1957;16:494-502.

10. Han SB, Lee DH, Shetty GM, Chae DJ, Song JG, Nha KW. A "safe zone" in medial open-wedge high tibia osteotomy to prevent lateral cortex fracture. Knee Surg Sports Traumatol Arthrosc. 2013;21:90-5.

11. Fujisawa Y, Masuhara K, Shiomi S. The effect of high tibial osteotomy on osteoarthritis of the knee: an arthroscopic study of 54 knee joints. Orthop Clin North Am. 1979;10:585608.

12. Schuster P, Schulz M, Mayer P, Schlumberger M, Immendoerfer M, Richter J. Open-wedge high tibial osteotomy and combined abrasion/microfracture in severe medial osteoarthritis and varus malalignment: 5-year results and arthroscopic findings after 2 years. Arthroscopy. 2015;31:127988.

13. Moreland JR, Bassett LW, Hanker GJ. Radiographic analysis of the axial alignment of the lower extremity. J Bone Joint Surg Am. 1987;69:745-9.

14. Bauer GC, Insall J, Koshino T. Tibial osteotomy in gonarthrosis (osteo-arthritis of the knee). J Bone Joint Surg Am. 1969;51:1545-63.

15. Pandit H, Jenkins C, Barker K, Dodd CA, Murray DW. The Oxford medial unicompartmental knee replacement using a minimally-invasive approach. J Bone Joint Surg Br. 2006;88: 54-60.

16. Brandon ML, Haynes PT, Bonamo JR, Flynn MI, Barrett GR, Sherman MF. The association between posterior-inferior tibial slope and anterior cruciate ligament insufficiency. Arthroscopy. 2006;22:894-9.

17. Tegner Y, Lysholm J. Rating systems in the evaluation of knee 
ligament injuries. Clin Orthop Relat Res. 1985;(198):43-9.

18. Lasam MP, Lee KJ, Chang CB, Kang YG, Kim TK. Femoral lateral bowing and varus condylar orientation are prevalent and affect axial alignment of TKA in Koreans. Clin Orthop Relat Res. 2013;471:1472-83.

19. Kim HA, Kim S, Seo YI, Choi HJ, Seong SC, Song YW, Hunter D, Zhang Y. The epidemiology of total knee replacement in South Korea: national registry data. Rheumatology (Oxford). 2008;47:88-91.

20. Koh IJ, Kim MW, Kim JH, Han SY, In Y. Trends in high tibial osteotomy and knee arthroplasty utilizations and demographics in Korea from 2009 to 2013. J Arthroplasty. 2015; 30:939-44.

21. Spahn G, Hofmann GO, von Engelhardt LV, Li M, Neubauer $\mathrm{H}$, Klinger HM. The impact of a high tibial valgus osteotomy and unicondylar medial arthroplasty on the treatment for knee osteoarthritis: a meta-analysis. Knee Surg Sports Traumatol Arthrosc. 2013;21:96-112.

22. Floerkemeier S, Staubli AE, Schroeter S, Goldhahn S, Lobenhoffer P. Outcome after high tibial open-wedge osteotomy: a retrospective evaluation of 533 patients. Knee Surg Sports Traumatol Arthrosc. 2013;21:170-80.

23. Pascale W, Luraghi S, Perico L, Pascale V. Do microfractures improve high tibial osteotomy outcome? Orthopedics. 2011; 34:e251-5.

24. Jung WH, Takeuchi R, Chun CW, Lee JS, Jeong JH. Comparison of results of medial opening-wedge high tibial oste- otomy with and without subchondral drilling. Arthroscopy. 2015;31:673-9.

25. Matsunaga D, Akizuki S, Takizawa T, Yamazaki I, Kuraishi J. Repair of articular cartilage and clinical outcome after osteotomy with microfracture or abrasion arthroplasty for medial gonarthrosis. Knee. 2007;14:465-71.

26. Harris JD, McNeilan R, Siston RA, Flanigan DC. Survival and clinical outcome of isolated high tibial osteotomy and combined biological knee reconstruction. Knee. 2013;20: 154-61.

27. Sterett WI, Steadman JR, Huang MJ, Matheny LM, Briggs KK. Chondral resurfacing and high tibial osteotomy in the varus knee: survivorship analysis. Am J Sports Med. 2010; 38:1420-4.

28. Hernigou P, Roussignol X, Flouzat-Lachaniette CH, Filippini P, Guissou I, Poignard A. Opening wedge tibial osteotomy for large varus deformity with Ceraver resorbable beta tricalcium phosphate wedges. Int Orthop. 2010;34:191-9.

29. Laprade RF, Spiridonov SI, Nystrom LM, Jansson KS. Prospective outcomes of young and middle-aged adults with medial compartment osteoarthritis treated with a proximal tibial opening wedge osteotomy. Arthroscopy. 2012;28:35464.

30. Bode G, von Heyden J, Pestka J, Schmal H, Salzmann G, Sudkamp N, Niemeyer P. Prospective 5-year survival rate data following open-wedge valgus high tibial osteotomy. Knee Surg Sports Traumatol Arthrosc. 2015;23:1949-55. 